This item was submitted to Loughborough's Research Repository by the author.

Items in Figshare are protected by copyright, with all rights reserved, unless otherwise indicated.

\title{
Cauchy problem for integrable discrete equations on quad-graphs
}

PLEASE CITE THE PUBLISHED VERSION

LICENCE

CC BY-NC-ND 4.0

\section{REPOSITORY RECORD}

Adler, V.E., and A.P. Veselov. 2019. "Cauchy Problem for Integrable Discrete Equations on Quad-graphs". figshare. https://hdl.handle.net/2134/257. 


\title{
Cauchy problem for integrable discrete equations on quad-graphs
}

\author{
Dedicated to S.P. Novikov \\ on his 65 birthday
}

\author{
V.E. Adler* A.P. Veselov ${ }^{\dagger}$
}

\begin{abstract}
Initial value problems for the integrable discrete equations on quadgraphs are investigated. We give a geometric criterion of when such a problem is well-posed. In the basic example of the discrete KdV equation an effective integration scheme based on the matrix factorization problem is proposed and the interaction of the solutions with the localized defects in the regular square lattice are discussed in details. The examples of kinks and solitons on various quad-graphs, including quasiperiodic tilings, are presented.
\end{abstract}

\section{Introduction}

The discrete potential $\mathrm{KdV}(\mathrm{dKdV})$ equation

$$
\left(v_{m+1, n+1}-v_{m, n}\right)\left(v_{m+1, n}-v_{m, n+1}\right)=\alpha_{m}-\beta_{n}, \quad m, n \in \mathbb{Z}
$$

can be considered as a simplest representative of the integrable nonlinear discrete equations in two dimensions. This field is widely studied and many other examples can be found in the literature, see e.g. [1 -7]. However, we

\footnotetext{
*Institut für Mathematik, Technische Universität Berlin, Str. des 17. Juni 136, 10623 Berlin, Germany. On leave from Landau Institute for Theoretical Physics, Chernogolovka, Russia. E-mail:adler@itp.ac.ru

${ }^{\dagger}$ Loughborough University, Loughborough, Leicestershire LE11 3TU, UK and Landau Institute for Theoretical Physics, Russia. E-Mail: A.P.Veselov@lboro.ac.uk
} 
restrict ourselves to this simplest model, since our aim here is to analyze the generalizations of another kind.

More precisely, we will discuss the role of the support of the discrete equations, that is, roughly speaking, the set where the independent variables live. Of course, the most natural way to discretize 2-dimensional PDE is to consider equations on the regular square grid $\mathbb{Z}^{2}$ as above, but recent results [ $8-15]$ demonstrate that possibly more general synonym of ' $2 \mathrm{D}$ ' in the discrete case is 'planar graphs'.

It should be noted that non-standard planar graphs were considered in Mathematical Physics already quite a while ago (see e.g. Korepin's works $[18,19]$ where the solvable spin models on the quasi-crystallic tilings were investigated). A systematic theory of the linear difference operators on the graphs in relation to soliton theory has been initiated by S.P. Novikov (see $[13-17])$.

We start in Section 2 with the necessary information about integrable equations on quad-graphs, which are planar graphs with quadrilateral faces. In this case the integrability can be understood as the so called 3D consistency property, introduced and studied in [9-12]. The discrete potential KdV equation on quad-graph reads locally exactly as on the square lattice:

$$
\left(v_{12}-v\right)\left(v_{1}-v_{2}\right)=\alpha_{1}-\alpha_{2},
$$

but now it is assumed that the fields $v$ are assigned to the vertices of the quad-graph and the parameters $\alpha$ are assigned to the edges, as shown on the Fig. 1 below, and the relation (2) must be fulfilled for each face of the graph.

Section 3 is devoted to the general discussion of the possible settings of Cauchy problem or initial value problem (IVP) on quad-graphs. At the first sight the question about the well-posedness of IVP is not related to integrability but in fact it does very much (see e.g. Fig. 10, 12 below). The 3D consistency property plays an essential role here.

Section 4 contains the main result of the paper: a criterion for the existence and uniqueness of the solution of Cauchy problem for an integrable equation on a quad-graph (Theorem 2).

In section 5 we discuss the effect of interaction of the solutions of the $\mathrm{dKdV}$ equation with the localized defects in the regular square lattice. We introduce a notion of the weak defect and show that such a defect does not affect the dynamics outside it. An integration scheme based on the matrix factorization problem plays a crucial role in the proof.

Several examples of the kink/solitons solutions on various quad-graphs, including quasiperiodic tilings are presented in the section 6 .

In the last section we discuss the solutions of the linear discrete wave 
equation

$$
v_{12}-v_{1}-v_{2}+v=0
$$

on quad-graphs. Surprisingly enough, in contrast to the non-linear case these solutions have a non-trivial interaction with weak defects, although the effect is limited (see Theorem 5).

\section{Quad-graph equations}

We start with the results of the paper [12] where all the integrable cases were classified among the certain class of discrete equations on quad-graphs. The basic building block of such equation is an equation on the elementary quadrilateral of the form

$$
Q\left(v, v_{1}, v_{2}, v_{12} ; \alpha_{1}, \alpha_{2}\right)=0
$$

where the field variables $v \in \mathbb{C}$ are assigned to the vertices and the parameters $\alpha \in \mathbb{C}$ are assigned to the edges of quadrilateral, as shown on the Fig. 1. The following properties are assumed to be satisfied:

1) the parameters $\alpha$ on the opposite edges of any face are equal;

2 ) the equation (3) is invariant under the group $D_{4}$ of the square symmetries:

$$
\begin{aligned}
& Q\left(v, v_{1}, v_{2}, v_{12} ; \alpha_{1}, \alpha_{2}\right)=\varepsilon Q\left(v, v_{2}, v_{1}, v_{12} ; \alpha_{2}, \alpha_{1}\right) \\
& =\sigma Q\left(v_{1}, v, v_{12}, v_{2} ; \alpha_{1}, \alpha_{2}\right), \quad \varepsilon, \sigma= \pm 1
\end{aligned}
$$

3) the function $Q$ is a polynomial of degree one with respect to each field variable.

The first property means that the value of parameter is constant along any strip (or, characteristic), which is a sequence of quadrilaterals adjacent

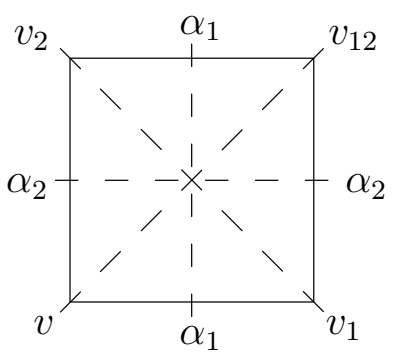

Figure 1: An elementary quadrilateral and its $D_{4}$ symmetry 


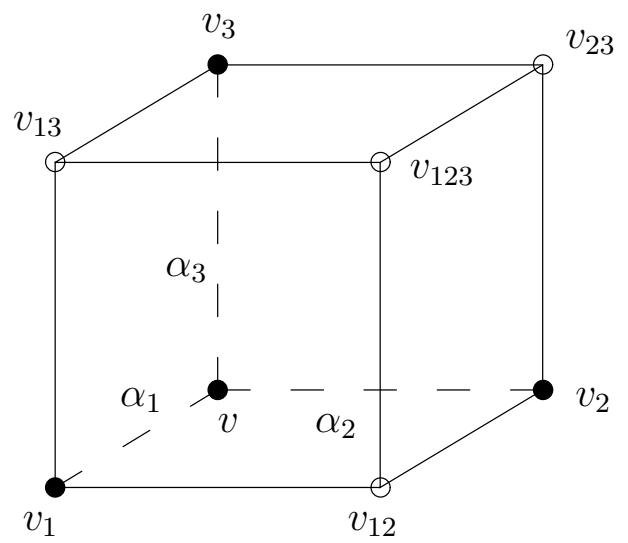

Figure 2: Three-dimensional consistency

by the opposite edges. We will see in the next sections that this notion is very important in the theory of quad-graph equations and plays the role similar to the characteristics for the second-order hyperbolic eqautions. In the particular case of $\mathrm{dKdV}$ equation on the square lattice (1) we have two families of the parameters $\alpha_{m}$ and $\beta_{n}$ assigned to the vertical and horizontal straight strips, but in the general case the strips may bend and intersect in a very complicated way (for instance, self-crossing, self-tangent and closed strips are possible). Some characteristics are shown at the pictures below by dashed lines crossing the adjacent edges of the faces in the strip.

The second property allows us to define equation on each face independently on its position in the quad-graph.

The third property means that the equation (3) can be solved with respect to a field at any vertex as linear fractional expression of other three fields. This expression could be singular for some special values of the fields, which is not essential for many types of quad-graphs, since we are interested only in the generic initial data. However, this becomes important for the graphs containing self-crossing strips, as we will see in Example 5.

The notion of integrability laid in the basis of the classification in [12] is the 3 -dimensional consistency $[9,10,11]$ which means that the equation (3) may be consistently embedded into a 3-dimensional lattice, so that the similar equations hold for all six faces of any elementary cube, as on Fig. 2. It should be mentioned that this property is in a close relation to the settheoretical solutions of the Yang-Baxter equation [20], or Yang-Baxter maps [21] (see the discussion of this in the Conclusions of [12]).

To describe more precisely what does the 3D consistency mean, consider 
the Cauchy problem with the initial data $v, v_{1}, v_{2}, v_{3}$. The equations on the rear faces

$$
Q\left(v, v_{i}, v_{j}, v_{i j} ; \alpha_{i}, \alpha_{j}\right)=0, \quad 1 \leq i<j \leq 3
$$

allow one to determine uniquely the values $v_{12}, v_{13}, v_{23}$. After that one has three different equations for $v_{123}$, coming from the front faces, and consistency means that all three values thus obtained for $v_{123}$ coincide. For example, in the discrete $\mathrm{KdV}$ case we have the formula

$$
v_{123}=\frac{\left(\alpha_{1}-\alpha_{2}\right) v_{1} v_{2}+\left(\alpha_{3}-\alpha_{1}\right) v_{3} v_{1}+\left(\alpha_{2}-\alpha_{3}\right) v_{2} v_{3}}{\left(\alpha_{3}-\alpha_{2}\right) v_{1}+\left(\alpha_{1}-\alpha_{3}\right) v_{2}+\left(\alpha_{2}-\alpha_{1}\right) v_{3}}
$$

independently on the order of calculations.

It should be noted that the variable $v$ does not appear in this expression. This property is very important in many aspects, in particulary in the classification problem which was solved in [12] only under this assumption. The role of this property for the Cauchy problems is not very clear. Although we will not use it in our analysis of the IVP below we will see that some results for the linear wave equation (which satisfies all the properties 1)-3) but not the $v$-independence) and for the equations from the list [12] will be different. Of course, linear equation is exceptional from many points of view, so it does not mean that this is related to this particular property.

The following Theorem is the immediate consequence of 3D consistency. It defines the Bäcklund transformation for quad-graph equation, and we will make use of it in the next Section.

Theorem 1. Let variables $v_{i}, i \in V(\Gamma)$ satisfy $3 D$ consistent quad-graph equation (3) on the quad-graph $\Gamma$. Let us fix some vertex $i_{0}$ and choose arbitrarily the values $\bar{v}_{i_{0}}$ and $\alpha$. Then the formulae

$$
Q\left(v_{i}, v_{j}, \bar{v}_{i}, \bar{v}_{j} ; \alpha_{i j}, \alpha\right)=0, \quad(i, j) \in E(\Gamma)
$$

define uniquely new solution $\bar{v}_{i}$ of equation (3).

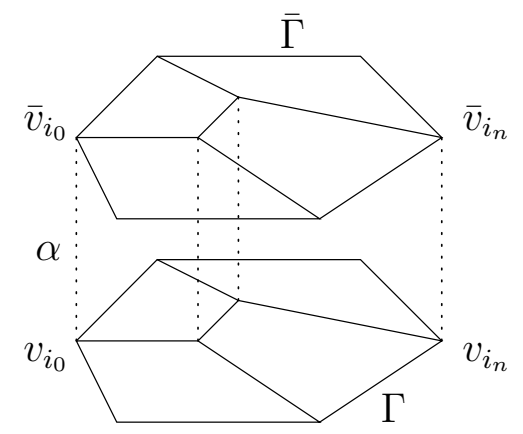

Figure 3: Bäcklund transformation 
Proof. Let us consider some path from $i_{0}$ to the given vertex $i_{n}$. Along this path we have the sequence of equations of the form

$$
Q\left(v_{i_{m}}, v_{i_{m+1}}, \bar{v}_{i_{m}}, \bar{v}_{i_{m+1}} ; \alpha_{i_{m}, i_{m+1}}, \alpha\right)=0, \quad m=0, \ldots, n-1
$$

which define recurrently $\bar{v}_{i_{1}}, \ldots, \bar{v}_{i_{n}}$. The fact that the different choice of the path gives the same result and that the constructed $\bar{v}_{i}$ solve (3) follows from the $3 \mathrm{D}$ consistency.

As it was observed in $[9,10,11]$ there is a direct link between the Bäcklund transformation (7) and the discrete zero curvature (or Lax) representation for the equation (3). We will call the matrix $L(x, y ; \alpha, \lambda)$ the Lax matrix for the equation (3) if this equation is equivalent to the relation

$$
L\left(v_{1}, v_{12} ; \alpha_{2}, \lambda\right) L\left(v, v_{1} ; \alpha_{1}, \lambda\right)=L\left(v_{2}, v_{12} ; \alpha_{1}, \lambda\right) L\left(v, v_{2} ; \alpha_{2}, \lambda\right)
$$

for any value of the spectral parameter $\lambda$ (cf. $[8,9]$ ).

The general procedure $[9,10,11]$ allows to construct for $3 \mathrm{D}$ consistent equations $2 \times 2$ Lax matrices in the following way. Put $\alpha=\lambda$ and rewrite the formula (7) in the form of the discrete Riccati equation

$$
\bar{v}_{j}=R\left(\bar{v}_{i}\right), \quad(i, j) \in E(\Gamma),
$$

where the function $R$ is linear fractional due to the property 3 ), with the coefficients depending on $v_{i}, v_{j}, \alpha_{i j} . \lambda$ This equation can be linearized by the substitution $\bar{v}=\psi / \phi$, which gives us the Lax matrix. In the discrete $\mathrm{KdV}$ case this procedure brings to the following matrices $L_{m, n}=L\left(v_{n}, v_{m} ; \alpha_{m n}, \lambda\right)$ :

$$
L_{m, n}=\left(\begin{array}{cc}
-v_{n} & v_{n} v_{m}+\alpha_{m n}-\lambda \\
-1 & v_{m}
\end{array}\right)
$$

We will use these matrices to investigate the IVP for the $\mathrm{dKdV}$ equation in the section 5 .

\section{Initial value problems}

Let us consider the initial value problem (or Cauchy problem) for a discrete equation on a quad-graph $\Gamma$ with initial data on a connected subgraph $P$. In most of the examples we consider $P$ is a simple path which is a connected sequence of the edges of the graph without self-intersections.

Slightly abusing terminology we say that such a Cauchy problem is wellposed if for the generic initial data the solution exists and unique (without 
investigation of the stability question). For finite graphs this is certainly justified since the stability is automatic then.

Let us first compare the number of equations and unknowns for the given quad-graph $\Gamma$. We assume that $\Gamma$ is simply connected and finite, and contains $F$ faces and $V$ vertices, from which $V_{b}$ ones belong to the boundary. Now apply the double counting of the angles in all faces (we assume for simplicity that each edge is a straight line, which, of course, is not necessary; the Euler formula provides the universal method). Since the sum of angles in each quadrilateral is equal to $2 \pi$, hence the total sum is equal to $2 \pi F$. On the other hand, summation over the vertices gives $2 \pi\left(V-V_{b}\right)$ for the interior nodes plus $\pi\left(V_{b}-2\right)$ for the boundary, so that the following equality holds:

$$
F=V-\frac{1}{2} V_{b}-1
$$

This means that in order to balance the number of equations and unknowns one has to to assign initial data in some $\frac{1}{2} V_{b}+1$ vertices. These vertices are not necessarily lie on the boundary, as the simplest example of a staircase in rectangular domain of the square lattice demonstrates (see Fig. 4; here and everywhere initial data are marked by solid line).
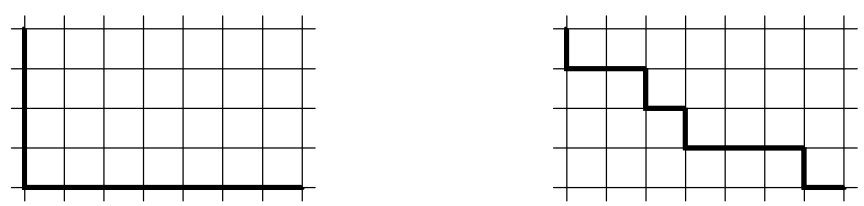

Figure 4: Possible choices of initial data on the square grid

Remark. The same calculation in the case of planar graph with $k$-lateral faces yields the formula $(k-2) F=2 V-V_{b}-2$. This means (under the natural assumption that $V_{b}<<V$ for large domains) that the systems on the graphs with triangular faces are overdetermined, while those on the graphs with $k>4$ are underdetermined. Of course, the balance between equations and unknowns can be restored in many ways. For example, one can consider systems with variable number of equations per face. In this way some integrable models were obtained on the triangular and hexagonal lattices, see e.g. [22]. Another possibility is to consider graphs with various types of the faces. 
Of course the above consideration is too rough and cannot answer how to choose the correct settings of Cauchy problem even in the simplest situations. In order to get some experience let us consider several examples assuming that equation (3) satisfies the properties 1)-3) from the previous Section, but is not necessarily integrable at first.

A priori several cases are possible: the IVP may be well-posed, or it may be underdetermined or overdetermined, and the calculation scheme for the well-posed IVP may be explicit (need only solving of one basic equation (3) at each step) or implicit (need to solve the systems of such equations). It turns out that all these possibilities can be easily realized on very simple quad-graphs.

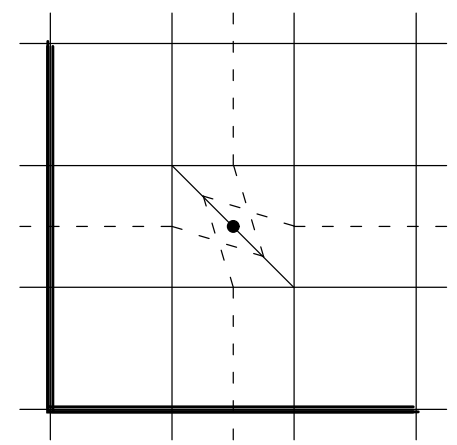

a

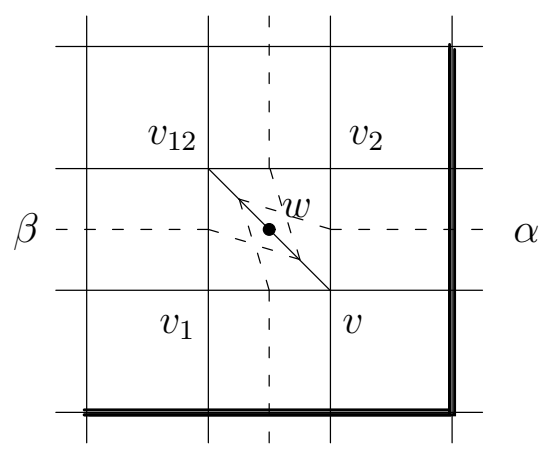

$\mathrm{b}$

Figure 5: Well-posed and overdetermined Cauchy problems

Example 1. It is easy to see that Cauchy problem shown on the Fig. 5a is well-posed, that is all fields are uniquely defined for arbitrary initial data (except for possible singularities which may arize in $\mathrm{dKdV}$ equation due to zero denominator). In contrast, the IVP on the right picture is not wellposed. Indeed, the values $v, v_{1}, v_{2}$ are uniquely defined, but for the values $w, v_{12}$ we have the system of two equations (use symmetry (4)):

$$
Q\left(v, v_{1}, w, v_{12} ; \alpha, \beta\right)=0, \quad Q\left(v, v_{2}, w, v_{12} ; \alpha, \beta\right)=0
$$

which gives the contradiction in the generic case $v_{1} \neq v_{2}$.

Example 2. Both IVP shown on the Fig. 6 are well-posed. For the left one this is obvious; for the right one we have the system of three equations on the variables $u, w, v_{12}$ :

$Q\left(v, v_{1}, u, v_{12} ; \alpha, \beta\right)=0, \quad Q\left(v, w, u, v_{12} ; \alpha, \beta\right)=0, \quad Q\left(v, w, v_{2}, v_{12} ; \alpha, \beta\right)=0$. 


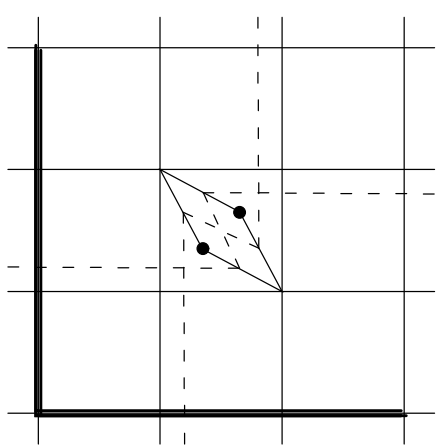

a

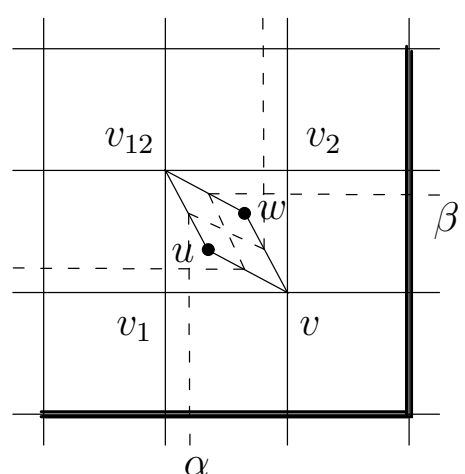

b

Figure 6: Well-posed Cauchy problems with explicit and implicit calculation scheme on transparent defect

This system implies $u=v_{2}, w=v_{1}$ and for $v_{12}$ one has the same equation $Q\left(v, v_{1}, v_{2}, v_{12} ; \alpha, \beta\right)=0$ as in the case of lattice without defect. In other words, the defect shown on these pictures does not affect the dynamics. We shall call defects with such property transparent.

Analogously the graph shown on the Fig. 7 gives explicit and implicit schemes depending on the choice of initial data.

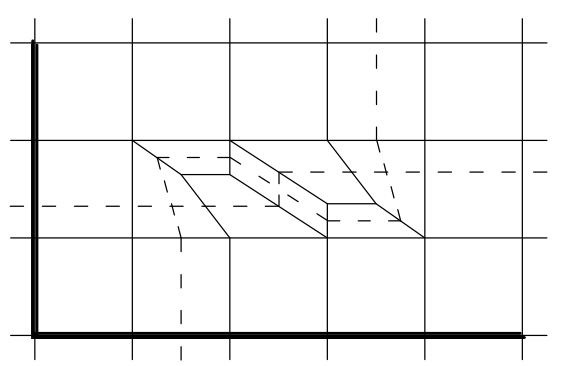

a

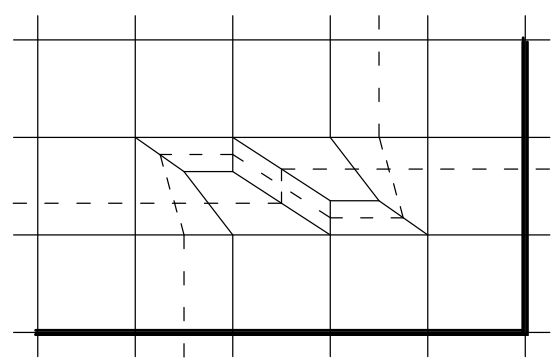

$\mathrm{b}$

Figure 7: Well-posed IVP with explicit (a) and implicit (b) schemes

Example 3. For the quad-graph shown on the Fig. 8 the first two IVP are not well-posed: for a) case one finds successfully the values $v$ inside the square bounded by initial data and the dashed characteristic, but encounters cotradiction outside it; for b) case the same square remains undetermined. 


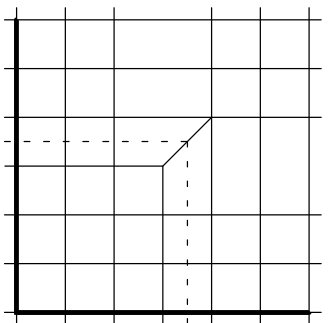

a

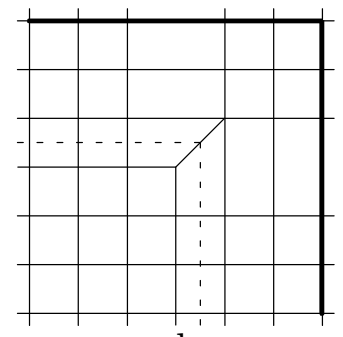

$\mathrm{b}$

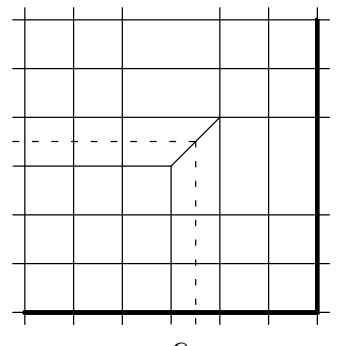

$\mathrm{C}$

Figure 8: Overdetermined, underdetermined and well-posed Cauchy problems

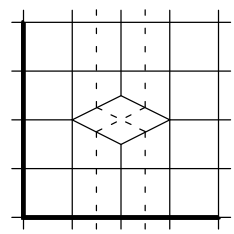

a
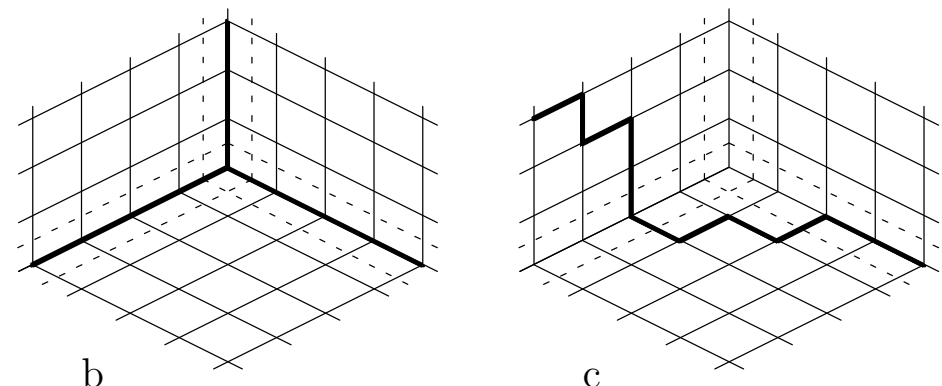

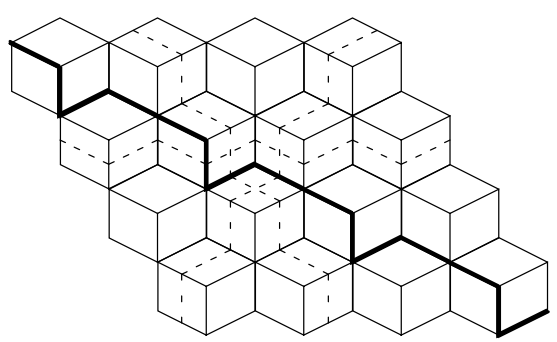

d

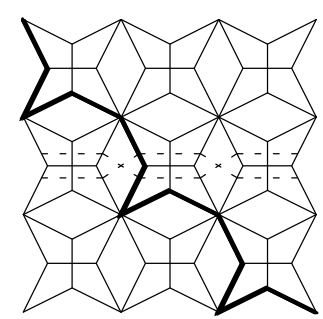

$\mathrm{e}$

Figure 9: Examples of well-posed Cauchy problems

Example 4. Several examples of well-posed IVP on different quad-graphs are shown on the Fig. 9.

Inspired by these examples and analogies with continuous case one could conjecture that an IVP with initial data given on a simple path $P$ is wellposed iff each characteristic (or strip) in $\Gamma$ meets $P$ exactly at one edge. We finish this section with two examples which show that for the general equation this conjecture is not true and emphasize the role of the 3D consistency condition in this problem. 


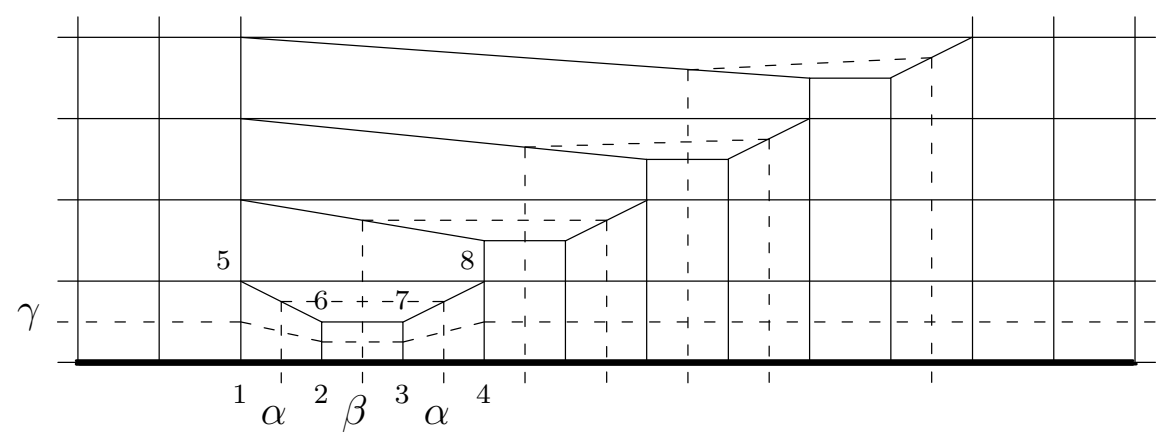

Figure 10: Well-posed/overdetermined Cauchy problem in general/integrable case.

Example 5. On the quad-graph on the Fig. 10, the vertical characteristics come back and cross the initial data again, while the horizontal characteristics do not cross the initial data at all. Nevertheless, this IVP is well-posed in general case, by means of implicit calculation scheme which can be described as follows:

1) the implicit step: unknowns $v_{5}, v_{6}, v_{7}, v_{8}$ are found as functions on $v_{1}, v_{2}$, $v_{3}, v_{4}$ by solving the system

$$
\begin{aligned}
& Q\left(v_{1}, v_{2}, v_{5}, v_{6} ; \alpha, \gamma\right)=0, \quad Q\left(v_{2}, v_{3}, v_{6}, v_{7} ; \beta, \gamma\right)=0 \\
& Q\left(v_{3}, v_{4}, v_{7}, v_{8} ; \alpha, \gamma\right)=0, \quad Q\left(v_{6}, v_{7}, v_{5}, v_{8} ; \beta, \alpha\right)=0 .
\end{aligned}
$$

2) next, all other variables on the bottom strip are computed explicitly;

3) now we are in the same situation and can repeat procedure.

Of course there is a question whether above system is degenerate or not. There is no reason for it to be degenerate for generic $Q$, but it turns out to be degenerate for $3 \mathrm{D}$ consistent equations. Indeed, in this case we can consider four quadrilaterals corresponding to the system (10), as the faces of $3 \mathrm{D}$ cube, and therefore the initial data $v_{1}, v_{2}, v_{3}, v_{4}$ must be related by equation $Q\left(v_{1}, v_{2}, v_{4}, v_{3} ; \alpha, \beta\right)=0$ in order to provide compatibility of this system. Moreover, even if this constraint is fulfilled, the values $v_{5}, v_{6}, v_{7}, v_{8}$ cannot be found uniquely, so in the integrable case this IVP is definitely not well-posed.

Example 6. The finite quad-graph on the Fig. 11 gives an example of the opposite situation: the Cauchy problem is well-posed for integrable equation and overdetermined for the generic one. In this example we have three characteristics, each crossing the path with initial data $v_{2}, v_{3}, v_{4}, v_{5}$ at one edge. 


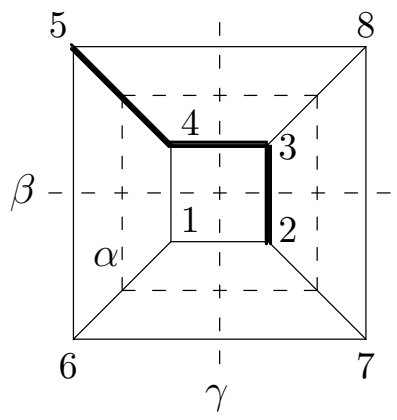

Figure 11: Overdetermined/well-posed Cauchy problem in general/integrable case.

The value $v_{8}$ can be computed in two ways: directly from the data $v_{3}, v_{4}, v_{5}$ or in several steps after finding consequently $v_{1}, v_{6}$ and $v_{7}$. In general, this values are different, so that the problem is overdetermined. However, for a $3 \mathrm{D}$ consistent equation both values coincide: the Fig. 11 is nothing but the projection of the cube on the Fig. 2.

\section{Existence and uniqueness theorem}

Now we are ready to formulate our main result.

Theorem 2. Let equation (3) be $3 D$ consistent, and let $P$ be a simple path in a finite simply connected planar quad-graph $\Gamma$ without self-intersecting strips. Consider Cauchy problem for this equation with generic initial data on the path $P$.

1) If each characteristic in $\Gamma$ intersects $P$ exactly at one edge then the IVP is well-posed, that is the solution exists and unique.

2) If some characteristic intersects P more than once then the IVP is overdetermined (no solution exists for generic initial data).

3) If some characteristic does not intersect $P$ then the IVP is underdetermined (if a solution exists, it is not unique).

We should clarify that when we consider the intersections of characteristics with a path we count only the internal edges of the characteristics. For example the intersection of a vertical characteristic with a staircase is one edge (but not two).

Before the proof let us comment on the self-intersecting strips. First of all their absence is essential for the statement as the example of the Cauchy problem for the $\mathrm{dKdV}$ equation on the quad-graph shown on the Fig. 12 


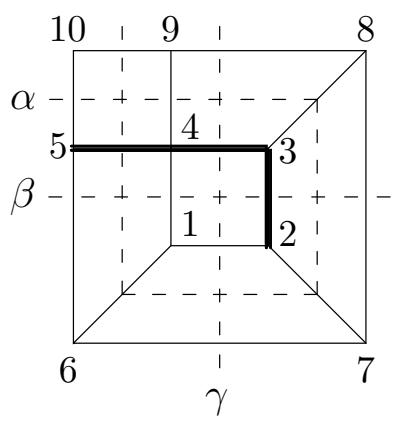

Figure 12: Self-intersecting strip: nonuniqueness in $v_{10}$

demonstrates. In this example the path with initial data $v_{2}, v_{3}, v_{4}, v_{5}$ crosses 3 characteristics; one computes consequently $v_{1}, v_{6}, v_{7}, v_{8}$ and $v_{9}=v_{5}$ automatically due to the $3 \mathrm{D}$ consistency. At this point the $\mathrm{dKdV}$ turns into $\left(v_{10}-v_{4}\right)\left(v_{9}-v_{5}\right)=0$, so that the value $v_{10}$ remains arbitrary.

However, in this case we can eliminate the self-intersecting strips from consideration by splitting them into the pairs of independent strips (one of them closed) by identification of the vertices in which the fields coincide, as shown on the Fig. 13. It should be noted that such splitting of the equation on the quadrilateral with the pair of equal parameters:

$$
Q\left(v, v_{1}, v_{2}, v_{12} ; \alpha, \alpha\right)=0 \quad \Rightarrow \quad\left(v_{12}-v\right)\left(v_{2}-v_{1}\right)=0
$$

takes place for all equations from the list obtained in the paper [12]. This means that for an integrable equation one can always eliminate all selfintersecting strips and thus reduce the problem to the graphs without such strips.

We would like to mention that the absence of self-crossing strips plays a crucial role in the general problem of embedding of a quad-graph into cubic lattice which was initiated by S.P. Novikov and investigated in details by Dolbilin et al [23]. In fact our proof of existence of solution is based on a
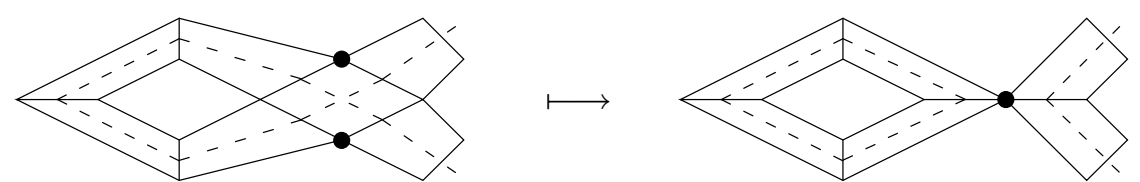

Figure 13: Elimination of self-intersecting strip 
similar geometric consideration, but instead of cubic lattice we consider a multidimensional cube.

Proof of existence. Assume that the conditions of the part 1) of the Theorem are fulfilled. Let us construct an immersion of $\Gamma$ into the $N$-dimensional unit cube, where $N$ is the length of $P$. To do this, assign the shift operators $T_{i}, i=1, \ldots, N$ to the edges of $P$. By definition, $T_{i}$ acts on $N$-dimensional vectors by formula $T_{i}(x)=x+e_{i}(\bmod 2)$ where $e_{i}$ is the vector with 1 at $i$-th place and 0 at the others.

Now distribute these operators at all edges of $\Gamma$ according to the same rule as for the parameters $\alpha$, i.e. one operator per strip. Obviously this can be done in unique way due to the condition 1 ). Choose the first vertex $v_{0}$ of $P$ as the origin with coordinates $(0, \ldots, 0)$ and define the coordinates of any other vertex $v$ as the result of applying shift operators along any path $P_{v}$ from $v_{0}$ to $v$. The result does not depend on the choice of $P_{v}$. Indeed, any closed path has an even intersection index with any characteristic and therefore is equivalent to identity operator.

Thus we have found an immersion of $\Gamma$ into $N$-dimensional unit cube and each face of $\Gamma$ now is just a 2 -dimensional face in this cube. Initial values are given on the path

$$
P=((0,0, \ldots, 0),(1,0, \ldots, 0),(1,1, \ldots, 0), \ldots,(1,1, \ldots, 1))
$$

and allow to calculate the values in each vertex of the cube. (It is not difficult to explain how to obtain the values for the coordinate vectors $e_{i}$, and so on.) Of course, 3D consistency is necessary here in order to justify the correctness of the procedure. In this way we construct some solution of the Cauchy problem.

Remark. It is worthy to note that all the fields at the vertices of the multidimensional cube can be calculated recursively using only one equation each time, so that on the cube the integration scheme becomes explicit. However, this algorithm of solving our Cauchy problem is not very effective, since the number $2^{N}$ of the vertices in the $N$-dimensional cube grows exponentially in $N$, while the number of vertices in $\Gamma$ (and therefore the size of the algebraic system we are solving) is about $N^{2}$.

The proof of the rest of the theorem presented below is based on the very different ideas and uses the notion of Bäcklund transformation (see Theorem 1). We will transform the initial quad-graph by erasing and inserting of a strip. These transformations bring, roughly speaking, to an equivalent IVP and can be described as follows. 


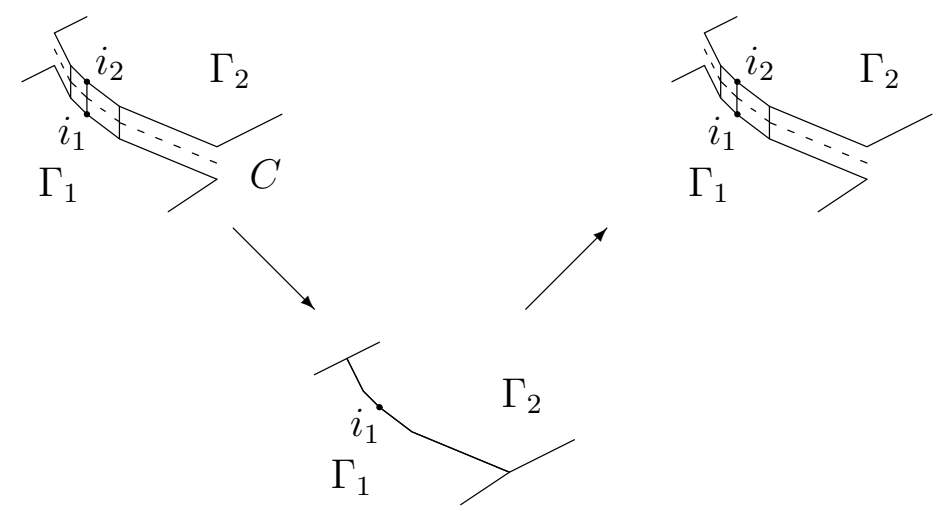

Figure 14: Erasing and inserting of a strip

Let us consider some strip $C$ with parameter $\alpha$. It divides $\Gamma$ into two subgraphs, $\Gamma_{1}$ and $\Gamma_{2}$. Let us denote $P_{i}$ the corresponding borders of the strip and let $i_{1} \in P_{1}$ and $i_{2} \in P_{2}$ be a pair of neighbour vertices separated by $C$. Now, let us apply BT (7) to the part $\Gamma_{2}$ of the quad-graph, taking $i_{2}$ as the seed point, and $\bar{v}_{i_{2}}=v_{i_{1}}$. This will give us some new solution in $\Gamma_{2}$ such that the variables $v$ along the border $P_{2}$ coincide with the corresponding variables $v$ along $P_{1}$. Therefore we can construct the common solution on the graph $\widetilde{\Gamma}$ obtained from $\Gamma$ by removing of the strip $C$. This solution coincides with the old one on $\Gamma_{1}$. Of course, some information is lost after such an operation and in order to make it invertible one have to remember the value $v_{i_{2}}$.

On the Fig. 14 these operations are shown for the case of simple strip, however the closed or self-tangent strips are allowed as well. For the selftangent strips the 3D consistency property implies the coincidence of the fields in some vertices, and these vertices can be merged during the above procedure (Fig. 15).
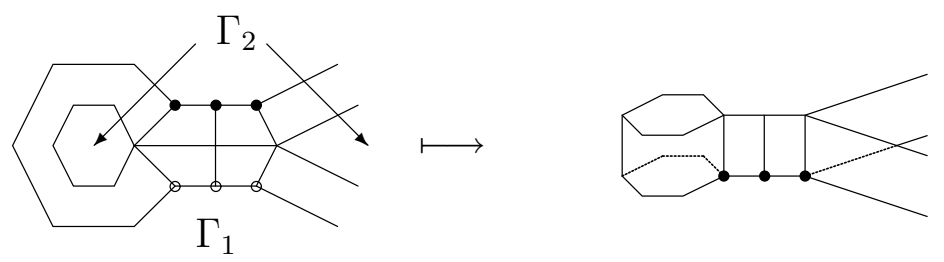

Figure 15: Case of self-tangent strip 
Proof of necessity. 2) Let some characteristic $C$ intersects the path $P$ with initial data twice. Let us enumerate the vertices of $P$ as shown on the Fig. 16, so that the edges of intersection are $(1,2)$ and $(n-1, n)$.
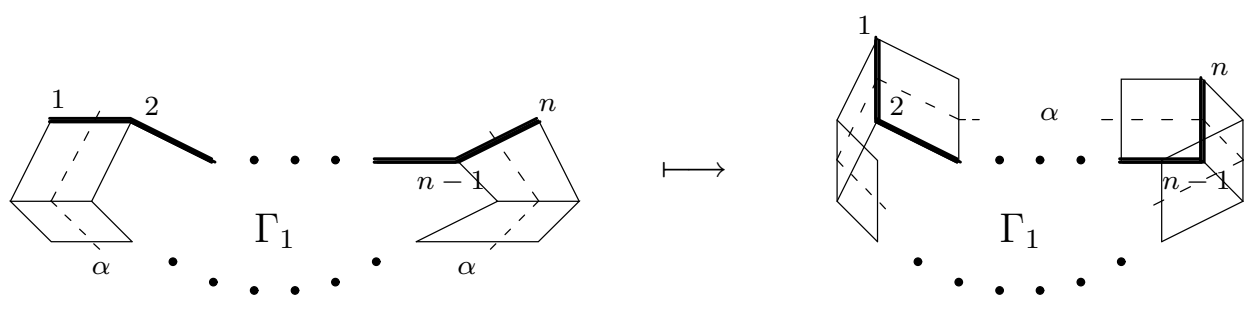

Figure 16: Constraint on the initial data

Suppose that IVP is well-posed, then solution of equation (3) exists on the subgraph $\Gamma_{1}$ bounded by $C$ and $P$. Let us look at this picture as 3dimensional one, assuming that the exterior border of the strip $C$ lies at the next floor, in particular the vertex 1 is over 2 and $n$ is over $n-1$. Accordingly to the Theorem 1 the value $v_{n}$ can be defined by solving the sequence of equation (7) along the path $(2, \ldots, n-1)$, that is $v_{n}$ is some function on $v_{1}, \ldots, v_{n-1}$. This means that the initial data are not free, that is the IVP is overdetermined.

2) Suppose that some strip $C$ never intersect the path $P$ with initial data. Let us cut off this strip, as shown on the Fig. 14 (we assume for defineteness that $P$ lies in the subgraph $\Gamma_{1}$ ). If a solution of the IVP exists in $\Gamma$ then this procedure gives us a solution with the same initial data on the reduced graph $\widetilde{\Gamma}$.

Next let us apply the inverse procedure to this solution, namely consider BT on $\Gamma_{2}$, taking some arbitrary value for $\bar{v}_{i_{1}}$. This gives us some solution on the graph $\Gamma$ which coincides with the original solution on $\Gamma_{1}$ but not on $\Gamma_{2}$. This means that the initial data are not sufficient to provide uniqueness of the solution.

Proof of uniqueness. This can be obtained by sequential erasing of the strips, as explained above, until the quad-graph is exhausted.

Remark. Although we have assumed that $P$ is a simple path (which is probably the most natural case), it follows from the proof that the result is true for any connected subgraph.

We will call a connected subgraph $P$ Cauchy subgraph if it has the property that each characteristic in $\Gamma$ meets $P$ exactly at one edge. 
A natural question is whether such a subgraph does always exist or not. The answer in general is negative. The following picture gives of an example of a regular lattice on which no well-posed IVP is possible, at least with initial data on the connected subgraphs. It is obtained from the kagome lattice by the standard process of merging with the dual graph (which is the quad-graph shown on the Fig. 9d). For the equations on quad-graphs of such type the question arise how to describe the set of general solutions.

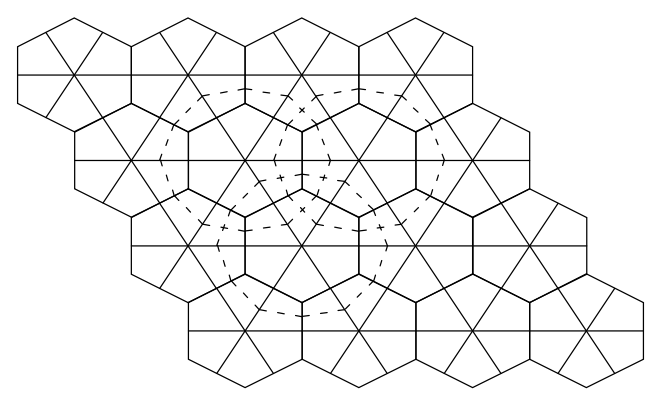

Figure 17: Example of transitive lattice without connected Cauchy subgraphs: all characteristics are closed

We would like to mention that as the example 6 above shows for a generic equation the Cauchy problem with the initial data on a Cauchy subgraph could not be well-posed. However in that example one of the characteristics is closed. We do not know if such an example exists without closed characteristics.

\section{Interaction of the solutions with the local- ized defects}

Consider the homogeneous $\mathrm{dKdV}$ equation

$$
\left(v_{m+1, n+1}-v_{m, n}\right)\left(v_{m+1, n}-v_{m, n+1}\right)=\alpha-\beta, \quad m, n \in \mathbb{Z}
$$

on the regular square lattice, assuming that all horizontal strips carry one and the same parameter $\alpha$ and analogously for the vertical strips.

Let us replace now some $m \times n$ rectangle by a finite quad-graph $D$ (with the same vertices on the boundary). What we will get is a regular square lattice with localized defect. Many examples of such localized defects can be 
found in the previous section, see e.g. Figures 5, 6, 7, 9a. Outside of the defect we have a regular picture of horizontal and vertical strips.

We will say that the defect is weak if all the characteristics entering the defect leave it in the same direction but possibly in a different order. One can see the examples of weak defects on the Figures 6, 7, 9a. On the Fig. 5 we have a non-weak defect since a pair of characteristics change their types.

It is clear that the homogeneous $\mathrm{dKdV}$ equation can be naturally defined on a regular lattice with a weak defect. Consider for the corresponding equation a Cauchy problem with the initial data outside the defect (e.g. on the staircases) just like for the usual square lattice. By the results of the previous Section for generic initial data it has a unique solution. Let us compare this solution with the solution of the same Cauchy problem but on the regular square lattice without defects.

The result turns out to be surprising: outside of the defect they will be the same.

Theorem 3. For the homogeneous dKdV equation a weak defect does not affect the generic solution outside the defect.

In other words for homogeneous $\mathrm{dKdV}$ solutions the weak defects are transparent. This is especially remarkable since for the discrete wave equation this is not true (see below Section 7).

The proof is based on the existence of discrete zero-curvature representation (8), (9) and follows from the following general considerations.

Let $P=\left(i_{1}, i_{2}, \ldots, i_{n}, i_{1}\right)$ be a closed path in a simply connected quadgraph $\Gamma$ then for any solution of $\mathrm{dKdV}$ equation on $\Gamma$ the ordered product of the Lax matrices (9) is equal to a scalar matrix. More precisely the formula

$$
\prod_{P}^{\curvearrowleft} L_{i_{k+1}, i_{k}}=\prod_{C}\left(\lambda-\alpha_{C}\right)^{\frac{1}{2} \operatorname{ind}(C, P)} I
$$

is valid where the product in the right hand side is taken over all characteristics in $\Gamma, \alpha_{C}$ is the parameter corresponding to the strip $C$ and the index $\operatorname{ind}(C, P)$ is the total number of intersections of $C$ and $P$ (without signs). This formula can be easily proved from the property (8) and the relation

$$
L_{i, j} L_{j, i}=\left(\lambda-\alpha_{i, j}\right) I .
$$

Equivalently, if $P_{1}=\left(i_{1}, i_{2}, \ldots, i_{m}\right)$ and $P_{2}=\left(j_{1}, j_{2}, \ldots, j_{n}\right)$ are two paths from $i_{1}=j_{1}$ to $i_{m}=j_{n}$ then

$$
\prod_{P_{1}}^{\curvearrowleft} L_{i_{k+1}, i_{k}}=\prod_{C}\left(\lambda-\alpha_{C}\right)^{\frac{1}{2}\left(\operatorname{ind}\left(C, P_{1}\right)-\operatorname{ind}\left(C, P_{2}\right)\right)} \prod_{P_{2}}^{\curvearrowleft} L_{j_{k+1}, j_{k}} .
$$


Theorem 4. Let the paths $P_{1}$ and $P_{2}$ have the common starting and end points and for any characteristic $C$ in $\Gamma$ we have ind $\left(C, P_{1}\right)=\operatorname{ind}\left(C, P_{2}\right) \leq 1$. Then for any generic solution of $d K d V$ equation on $\Gamma$ the fields $v$ on $P_{2}$ can be uniquely recovered from the values on $P_{1}$.

Proof. Let us consider the matrix product $L(\lambda)=\prod_{P_{1}}^{\curvearrowleft} L_{i_{k+1}, i_{k}}=\left(\begin{array}{ll}a & b \\ c & d\end{array}\right)$. One can prove that for a generic solution $\operatorname{rank} L\left(\alpha_{C}\right)=1$ for each strip $C$ intersecting $P_{1}$ (for some special solutions this can fail: for example if we have $\alpha_{i_{k+2}, i_{k+1}}=\alpha_{i_{k+1}, i_{k}}=\alpha$ and $v_{i_{k+2}}=v_{i_{k+1}}$ for three consequent vertices in the path, then $\left.\left.L_{i_{k+2}, i_{k+1}} L_{i_{k+1}, i_{k}}\right|_{\lambda=\alpha} \equiv 0\right)$.

Now the result follows from the uniqueness of the refactorization of the same matrix as $L(\lambda)=\prod_{P_{2}}^{\curvearrowleft} L_{j_{k+1}, j_{k}}$ along the path $P_{2}$. It is enough to show that there exists a unique matrix of the form

$$
L_{j_{2}, j_{1}}=\left(\begin{array}{cc}
-v_{j_{1}} & v_{j_{1}} v_{j_{2}}+\alpha_{j_{2}, j_{1}}-\lambda \\
-1 & v_{j_{2}}
\end{array}\right)
$$

such that $L(\lambda)=\tilde{L} L_{j_{2}, j_{1}}$ and the matrix $\tilde{L}$ is polynomial in $\lambda$. Indeed, $v_{j_{2}}$ is uniquely defined from the condition ker $L\left(\alpha_{j_{2}, j_{1}}\right) \sim\left(\begin{array}{c}v_{i_{2}} \\ 1\end{array}\right)$. Since the polynomials $a v_{i_{2}}+b$ and $c v_{j_{2}}+d$ are divisible by $\lambda-\alpha_{j_{2}, j_{1}}$, hence the matrix

$$
\tilde{L}=\frac{1}{\lambda-\alpha_{j_{2}, j_{1}}}\left(\begin{array}{ll}
-a v_{j_{2}}-b & \left(\alpha_{j_{2}, j_{1}}-\lambda\right) a+v_{j_{1}}\left(a v_{j_{2}}+b\right) \\
-c v_{j_{2}}-d & \left(\alpha_{j_{2}, j_{1}}-\lambda\right) c+v_{j_{1}}\left(c v_{j_{2}}+d\right)
\end{array}\right)
$$

is polynomial. Continuing in the same way we will reconstruct the solution on the path $P_{2}$.

Now to derive the Theorem 3 one should consider two sides of the rectangle $D$ directed towards the Cauchy subgraph as $P_{1}$ and other two sides as $P_{2}$.

Notice that in general the Cauchy problem with the initial data on $P_{1}$ may be underdetermined, since some characteristics may not intersect it. For example, some closed strips may occur inside the subgraph bounded by $P_{1}$ and $P_{2}$. Nevertheless, the use of transition matrices $L_{j i}$ will allow us to skip over such regions of underdeterminancy.

For a well-posed IVP the proof of Theorem 4 gives an integration scheme which allows to obtain the solution of $\mathrm{dKdV}$ equation by refactorization of the $L$ matrices product along the path of initial data. It is probably the most effective scheme one can suggest for this problem. 


\section{Kinks and solitons on quad-graphs}

In this section we consider some explicit solutions of $\mathrm{dKdV}$ equation rather than solutions of Cauchy problem. The hint is to search for solution compatible with the continuous dynamics. The $\mathrm{dKdV}$ equation on $\mathbb{Z}^{2}$ lattice (1) originates from the nonlinear superposition principle for the Bäcklund transformation

$$
\bar{v}_{x}+v_{x}=(\bar{v}-v)^{2}-\lambda
$$

of the potential KdV equation $v_{t}=v_{x x x}-6 v_{x}^{2}$. Usage of equation (12) allows to obtain the profile of the kink solution, and then to find algebraically the dependence of the phase on $m$ and $n$ (the dependence on $t$ can be easily recovered, but now this is unessential).

Let us start from the the simplest situation of constant parameters $\alpha_{m}=$ $\alpha, \beta_{n}=\beta$. In this case the linear seed solution is obvious

$$
v_{m, n}=a m+b n+p x+q, \quad a^{2}-b^{2}=\alpha-\beta .
$$

Applying the Bäcklund transformation (12) one obtains

$$
\bar{v}_{m, n}=a m+b n+p x+q-k \tanh \left(k x+\phi_{m, n}\right), \quad \lambda=2 p-k^{2} .
$$

Let us search for the dependence of $\phi$ on $m, n$ in the form

$$
\phi_{m, n}=\mu m+\nu n+\xi
$$

and denote

$$
e^{2\left(k x+\phi_{m, n}\right)}=X, \quad e^{2 \mu}=M, \quad e^{2 \nu}=N .
$$

Then substitution into (11) yields

$$
\left(a+b+k \frac{X-1}{X+1}-k \frac{M N X-1}{M N X+1}\right)\left(a-b+k \frac{N X-1}{N X+1}-k \frac{M X-1}{M X+1}\right)=a^{2}-b^{2} .
$$

Collecting together and splitting over $X$ give the relations between the parameters which can written in the form

$$
a \tanh \mu=b \tanh \nu=k,
$$

so that finally 1-kink solution is given by the formulae

$$
v_{m, n}=a m+b n+p x+q-k \tanh (k x+\mu m+\nu n+\xi),
$$

where

$$
a^{2}-b^{2}=\alpha-\beta, \quad \mu=\frac{1}{2} \log \frac{a+k}{a-k}, \quad \nu=\frac{1}{2} \log \frac{b+k}{b-k} .
$$




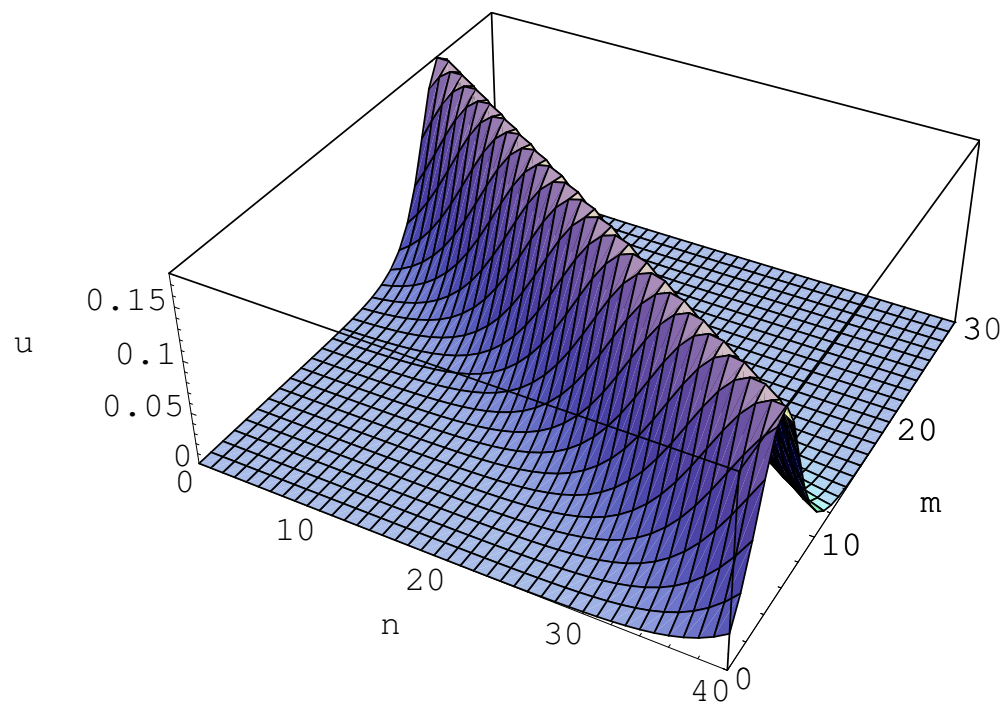

Figure 18: Soliton at $a=1, b=2, k=0.3, p=q=x=0, \xi=-7.5$

Obviously, one has to choose $|k|<\max \{|a|,|b|\}$ in order to obtain real $\mu, \nu$. The dependence on the parameter $x$ can be neglected, but we may also make use of it in order to obtain the discrete dynamics of the soliton solution $u_{m, n}=$ $-2 \partial_{x}\left(v_{m, n}\right)$ which corresponds to the usual KdV equation $u_{t}=u_{x x x}+6 u u_{x}$ (see Fig. 18).

One can check using the formula (12) that the corresponding fields $u$ satisfy the following discrete $K d V$ equation

$$
\sqrt{u_{12}+u_{2}-2 \alpha}-\sqrt{u_{1}+u-2 \alpha}=\sqrt{u_{12}+u_{1}-2 \beta}-\sqrt{u_{2}+u-2 \beta}
$$

or, equivalently,

$$
\begin{aligned}
& \frac{1}{4}\left(u_{12}-u\right)^{2}\left(u_{1}-u_{2}\right)^{2}-(\alpha-\beta)\left(u_{12}-u\right)\left(u_{1}-u_{2}\right)\left(u+u_{1}+u_{2}+u_{12}\right) \\
& +(\alpha-\beta)^{2}\left(\left(u_{12}-u\right)^{2}+\left(u_{1}-u_{2}\right)^{2}\right)+2\left(\alpha^{2}-\beta^{2}\right)\left(u_{12}-u\right)\left(u_{1}-u_{2}\right)=0 .
\end{aligned}
$$

In contrast to the discrete potential $\mathrm{KdV}$ equation this equation is quadratic with respect to each field, and thus is not covered by the classification [12].

Two-kink solution can be obtained via NSP for Bäcklund transformation, which brings to the formula

$$
v^{(12)}=v^{(0)}-\frac{k_{2}^{2}-k_{1}^{2}}{k_{2} \tanh \left(k_{2} x+\phi_{2}\right)-k_{1} \tanh \left(k_{1} x+\phi_{1}\right)}
$$


where the seed solution $v^{(0)}$ and phases $\phi_{1}, \phi_{2}$ are defined as above. In order to obtain profile without singularity in $x$ one may choose $k_{2}>k_{1}>0$ and make a shift $\phi_{2} \rightarrow \phi_{2}+\frac{\pi i}{2}$. In this way the general multikink solution can be constructed. Notice the different effect of the Bäcklund transform which may result either in the dressing of the solution or just in the phase shifts $m \rightarrow m+1, n \rightarrow n+1$.

The seed solution (13) is easily generalized for the case of variable parameters:

$$
v_{m, n}=a_{m}+b_{n}+p x+q, \quad\left(a_{m+1}-a_{m}\right)^{2}-\alpha_{m}=\left(b_{n+1}-b_{n}\right)^{2}-\beta_{n}=\delta .
$$

(Notice that this is more general ansatz even in the case of constant $\alpha, \beta$.) Proceeding as above one obtains the kink moving with the variable velocity:

$$
v_{m, n}=a_{m}+b_{n}+p x+q-k \tanh \left(k x+\mu_{m}+\nu_{n}+\xi\right),
$$

where

$$
\left(a_{m+1}-a_{m}\right) \tanh \left(\mu_{m+1}-\mu_{m}\right)=\left(b_{n+1}-b_{n}\right) \tanh \left(\nu_{n+1}-\nu_{n}\right)=k,
$$

that is

$$
\mu_{m+1}-\mu_{m}=\frac{1}{2} \log \frac{a_{m+1}-a_{m}+k}{a_{m+1}-a_{m}-k}, \quad \nu_{n+1}-\nu_{n}=\frac{1}{2} \log \frac{b_{n+1}-b_{n}+k}{b_{n+1}-b_{n}-k} .
$$

On the Fig. 19 the graph of the soliton $u=-2 v_{x}$ is plotted corresponding to the values

$$
\begin{gathered}
a_{0}=b_{0}=\mu_{0}=\nu_{0}=p=q=x=0, \\
a_{m}-a_{m-1}=\sqrt{m}, \quad b_{n}-b_{n-1}=\sqrt{2 n}, \quad k=0.3, \quad \xi=-3 .
\end{gathered}
$$

Moreover, the formula (17) can be easily generalized for the case of multidimensional lattice $\mathbb{Z}^{d}$ in which any $2 \mathrm{D}$ sublattice is governed by $\mathrm{dKdV}$ equation:

$$
v_{n_{1}, \ldots, n_{d}}=a_{n_{1}}^{1}+\cdots+a_{n_{d}}^{d}+p x+q-k \tanh \left(k x+\nu_{n_{1}}^{1}+\cdots+\nu_{n_{d}}^{d}+\xi\right),
$$

where

$$
\begin{gathered}
\left(a_{n+1}^{i}-a_{n}^{i}\right)^{2}-\alpha_{n}^{i}=\delta, \quad\left(a_{n+1}^{i}-a_{n}^{i}\right) \tanh \left(\nu_{n+1}^{i}-\nu_{n}^{i}\right)=k \\
\nu_{n+1}^{i}-\nu_{n}^{i}=\frac{1}{2} \log \frac{a_{n+1}^{i}-a_{n}^{i}+k}{a_{n+1}^{i}-a_{n}^{i}-k} .
\end{gathered}
$$




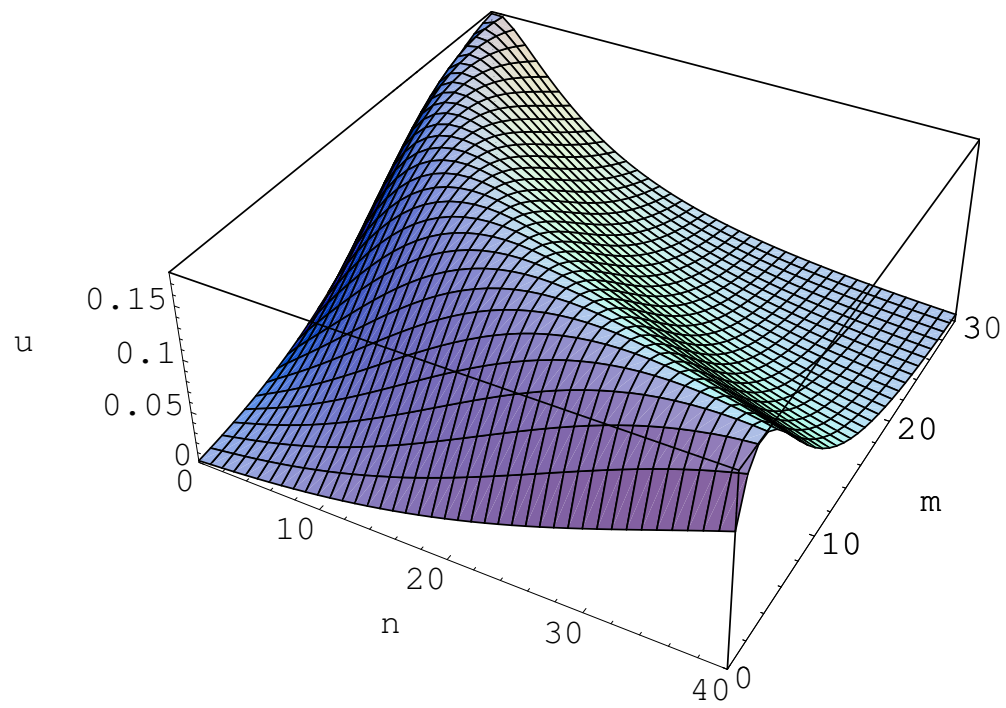

Figure 19: Bended soliton

This observation immediately gives us the kink solution for the quadgraphs which can be embedded into $\mathbb{Z}^{d}$ lattice. For instance, the graph shown on the Fig. 9d can be obtained from the cubic lattice by taking all its faces which intersect the plane $n_{1}+n_{2}+n_{3}=0$, so that the kink solution for this graph is given by the formula (18) at $d=3$ restricted on the values $n_{1}+n_{2}+n_{3} \in\{-1,0,1\}$. In the case of the linear phase $\nu_{n_{i}}^{i}=\nu^{i} n_{i}$ the corresponding soliton propagates in the direction $\left(\nu^{3}-\nu^{2}, \nu^{1}-\nu^{3}, \nu^{2}-\nu^{1}\right)$ (see Fig. 20).

Some subclass of the quasiperiodic tilings can be obtained if one choose the other planes and use de Bruijn's projection method (see e.g. [24]). The formula (18) works for such quad-graphs as well. On the Fig. 21 the same $3 \mathrm{D}$ solution is presented, restricted on the plane $n_{1}+\sqrt{2} n_{2}+\sqrt{3} n_{3}=0$.

Two more figures Fig. 22 and 23 present the 3-dimensional solution restricted on the coordinate quadrants.

An interesting problem is to investigate the interaction of the kink solutions with the localized defects in the square lattice. According to the Theorem 3 a weak defect in the homogeneous lattice does not affect solutions, so that the ordinary kink (14) does not change. In contrast, it can be shown that the bended kink (17) may acquire some phase shift after the interaction with such a defect. 


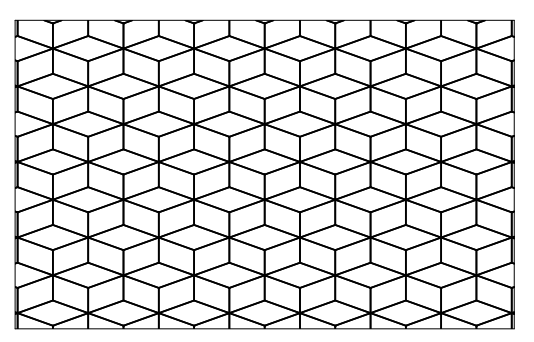

u

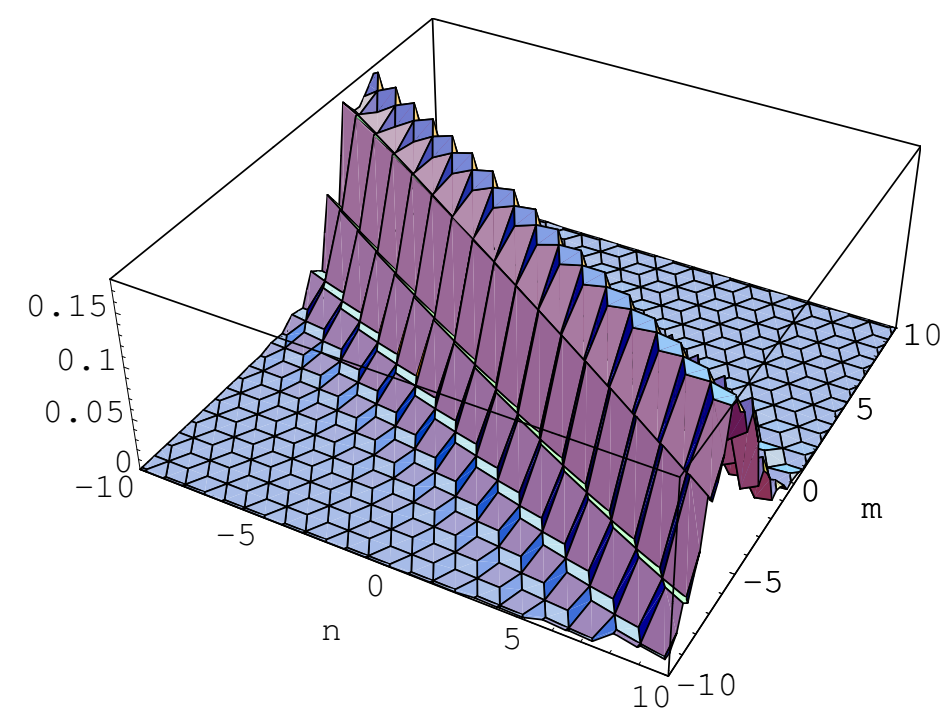

Figure 20: Soliton on the regular quadgraph $\left(a_{1}=0.5, a_{2}=2.5, a_{3}=3\right.$, $k=0.3, p=q=x=\xi=0$ ) 


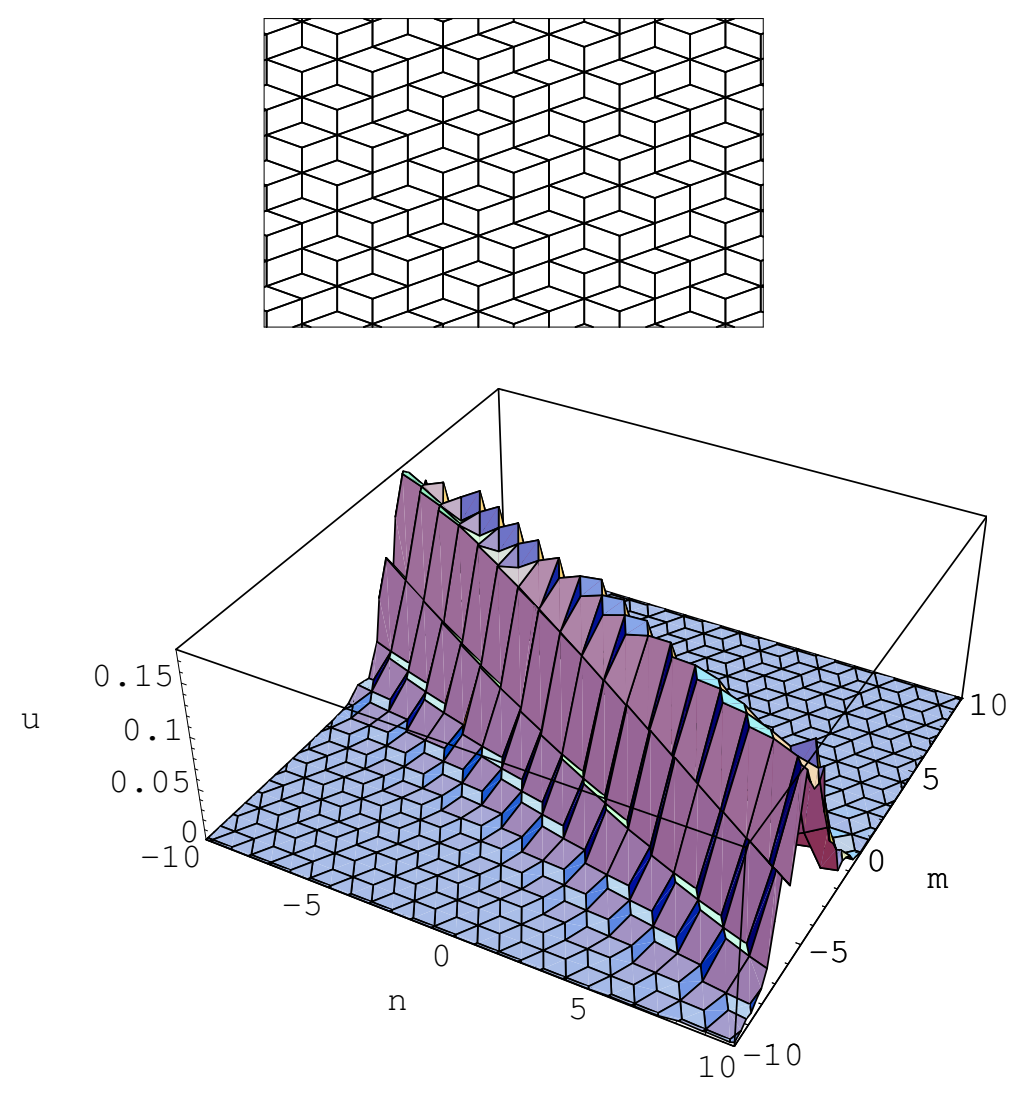

Figure 21: Soliton on the quasiregular quadgraph 

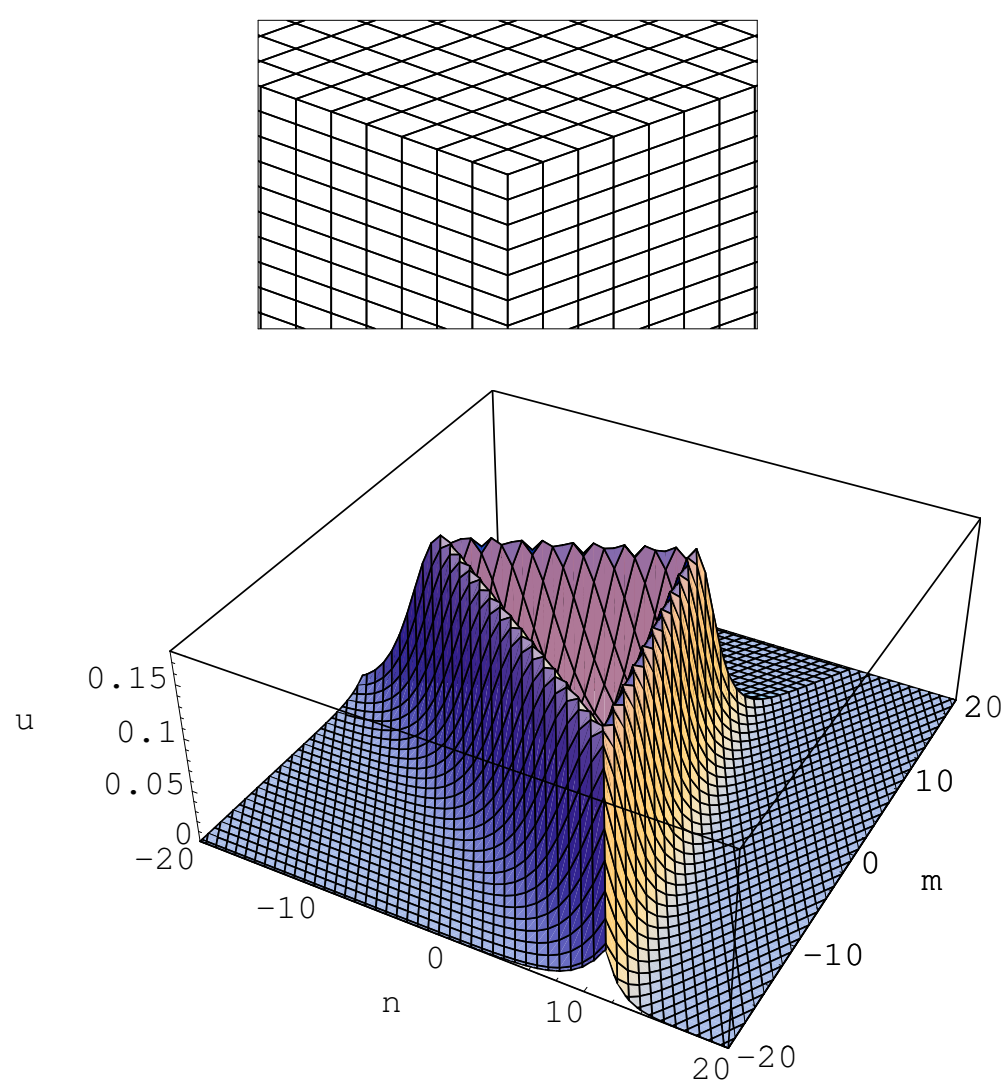

Figure 22: Soliton on three quadrants $n_{i}>0\left(a_{1}=1, a_{2}=1.5, a_{3}=2\right.$, $k=0.3, p=q=x=0, \xi=-3)$ 

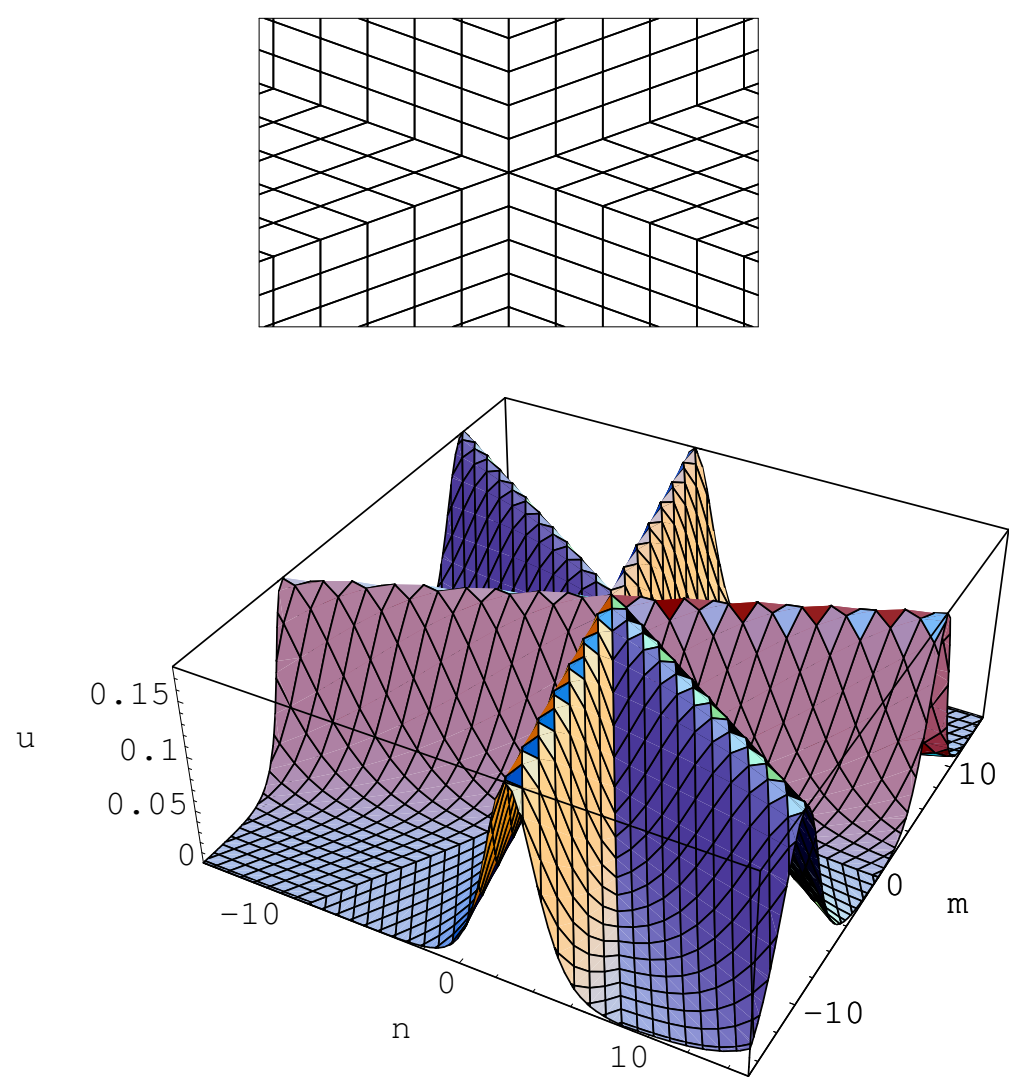

Figure 23: Soliton on six quadrants $n_{i} n_{j}<0\left(a_{1}=1, a_{2}=1.5, a_{3}=2\right.$, $k=0.3, p=q=x=\xi=0$ ) 


\section{Discrete linear wave equation}

It is interesting to compare the theory of the homogeneous dKdV equation we discussed above with the discrete linear wave equation

$$
v_{12}-v_{1}-v_{2}+v=0
$$

on quad-graphs.

Obviously, equation (19) always can be solved with respect to any vertex and it does not contain singularities in contrast to the $\mathrm{dKdV}$ case. Therefore the splitting of self-intersecting strips is not valid in this case.

The most serious distinction is that since the discrete wave equation does not contain the parameters the corresponding matrices (8)

$$
L_{j, i}=\left(\begin{array}{cc}
1 & v_{j}-v_{i} \\
0 & 1
\end{array}\right)
$$

contain no spectral parameter and give the fake zero curvature representation. In particular, the refactorization scheme from the Section 5 does not work, and the Theorem 3 is not valid, as the following simple examples demonstrate. Of course, for the wave equation it is sufficient to analyze the propagation of delta-like initial data (black circle denotes 1, white one denotes -1 , no circle denotes 0 ).

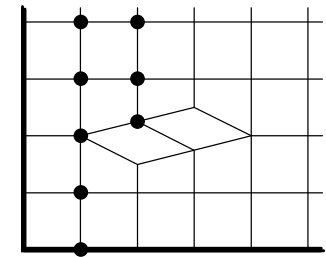

a

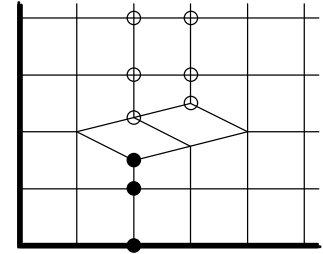

$\mathrm{b}$

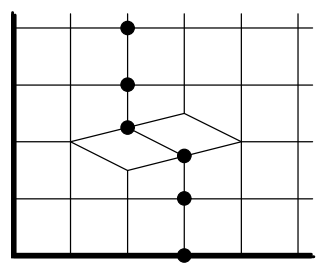

$\mathrm{C}$

Figure 24: Interaction of the linear wave with the weak defect: splitting, reflection, bending

Nevertheless we can claim that a weaker version of the theorem 3 is valid also for the discrete wave equation. Let us consider the Cauchy problem for this equation on a regular square lattice with a weak defect $D$ with initial data on a staircase (or coordinate half-axes) outside the defect. Again as in $\mathrm{dKdV}$ case according to our Theorem 2 the solution exists and unique and one can compare this solution with the one on the regular lattice without defects. 
Let us consider the cross $C(D)$ corresponding to the defect $D$ as the union of all the characterstics passing through $D$. Besides the defect itself it contains also all the vertical and horizontal strips passing through the rectangle.

Theorem 5. For the discrete wave equation a weak defect $D$ does not affect the solution outside the cross $C(D)$.

The proof easily follows from the fact that for the discrete wave equation the difference $v-v_{1}$ is preserved when we are moving along the corresponding charactersitic. Actually the affected area is only a half of this cross ("thick hook"; see the picture below). For a general equation one should expect the whole quadrant bounded by this hook to be affected.

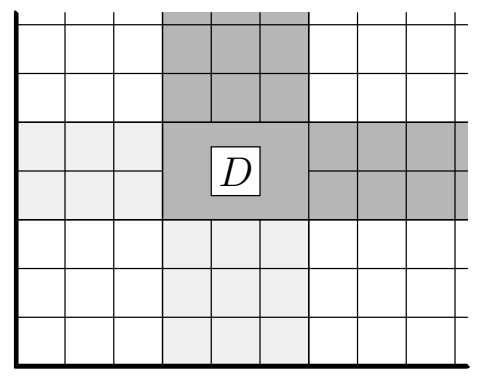

Figure 25: The area affected by the defect $D$ for the discrete linear wave equation

\section{Concluding remarks}

We have shown that for the integrable equations on the quad-graphs it is possible to formulate a criterion for the well-posedness of the Cauchy problems. The 3D consistency condition plays a crucial role in our analysis. The existence of Lax matrices provides an effective integration scheme for the corresponding IVP problems based on matrix factorization problem.

The question is whether such a criterion exists for a general equation on quad-graphs. One can easily produce a lot of examples of graphs and IVP which are not sensitive to a particular form of the equation, but to describe all of them in some geometric way seems to be a hard problem.

We have not discussed also global geometrical aspects (e.g. the quadgraphs on the surfaces of genus $g$ ). For some interesting results in this direction in the linear case we refer to Novikov and Dynnikov papers [13$16]$. 
The discrete equations in three-dimensions in the context of the classical geometry are investigated in the recent very interesting papers $[25,26,27]$ by B. Konopelchenko, W. Schief and A. King. In particular in [27] an example of a well-posed Cauchy problem for the discrete BKP equation is discussed.

Acknowledgments. The research by V.A. was partly supported by the Alexander von Humboldt Stiftung and by the RFBR grant 02-01-00144.

A.V. is grateful to S.P. Novikov who stimulated his interest to the problems on the graphs.

\section{References}

[1] L. Bianchi. Vorlesungen über Differenzialgeometrie. Teubner: Leipzig, 1899.

[2] R. Hirota. Nonlinear partial difference equations. I. A difference analog of the Korteweg-de Vries equation. III. Discrete sine-Gordon equation. J. Phys. Soc. Japan 43 (1977) 1423-1433, 2079-2086.

[3] T. Miwa. On Hirota's difference equations. Proc. Japan Acad., Ser. A: Math. Sci. 58:1 (1982) 9-12.

[4] G.R.W. Quispel, F.W. Nijhoff, H.W. Capel, J. van der Linden. Linear integral equations and nonlinear difference-difference equations. Physica A 125 (1984) 344-380.

[5] V.G. Papageorgiou, F.W. Nijhoff, H.W. Capel. Integrable mappings and nonlinear integrable lattice equations. Phys. Lett. A 147:2-3 (1990) 106114.

[6] H.W. Capel, F.W. Nijhoff, V.G. Papageorgiou. Complete integrability of Lagrangian mappings and lattices of KdV type. Phys. Lett. A 155 (1991) 377-387.

[7] F.W. Nijhoff, H.W. Capel. The discrete Korteweg-de Vries equation. Acta Appl. Math. 39 (1995) 133-158.

[8] V.E. Adler. Discrete equations on planar graphs. J. Phys. A: Math. Gen. 34 (2001) 10453-10460.

[9] A.I. Bobenko, Yu.B. Suris. Integrable systems on quad-graphs. Int. Math. Res. Notices 11 (2002) 573-611.

[10] F.W. Nijhoff, A.J. Walker. The discrete and continuous Painlevé hierarchy and the Garnier system. Glasgow Math. J. 43A (2001) 109-123. 
[11] F.W. Nijhoff. Lax pair for the Adler (lattice Krichever-Novikov) system. Phys. Lett. A 297 (2002), no. 1-2, 49-58.

[12] V.E. Adler, A.I. Bobenko, Yu.B. Suris. Classification of integrable equations on quad-graphs. The consistency approach. Comm. Math. Phys. 233 (2003), no. 3, 513-543.

[13] S.P. Novikov, I.A. Dynnikov. Discrete spectral symmetries of lowdimensional differential operators and difference operators on regular lattices and two-manifolds. Uspekhi Mat. Nauk 52:5 (1997) 175-234 [English translation: Russian Math. Surveys 52:5 (1997) 1057-1116].

[14] S.P. Novikov. The Schrödinger operators on graphs and topology. Uspekhi Mat. Nauk 52:6 (1997) 177-178 [English translation: Russian Math. Surveys 52:6 (1997) 1320-1321].

[15] I.A. Dynnikov, S.P. Novikov. Laplace transformations and simplicial connections. Uspekhi Mat. Nauk 52:6 (1997) 157-158 [English translation: Russian Math. Surveys 52:6 (1997) 1294-1295].

[16] I.A. Dynnikov, S.P. Novikov. Geometry of the triangle equation on twomanifolds. math-ph/0208041.

[17] I.M. Krichever, S.P. Novikov. Trivalent graphs and solitons. Uspekhi Mat. Nauk 54:1 (1999) 149-150 [English translation: Russian Math. Surveys 54:1 (1999) 1248-1249].

[18] V.E. Korepin. Exactly solvable spin models for quasicrystals. JETP 92:3 (1987) 1082-1089.

[19] V.E. Korepin. Completely integrable models in quasicrystals. Comm. Math. Phys. 110:1 (1987) 157-171.

[20] V.G. Drinfeld. On some unsolved problems in quantum group theory. In "Quantum groups" (Leningrad, 1990), Lecture Notes in Math., 1510, Springer, 1992, p. 1-8.

[21] A.P. Veselov. Yang-Baxter maps and integrable dynamics. Phys. Lett. A 314 (2003) 214-221.

[22] A.I. Bobenko, T. Hoffmann, Yu.B. Suris. Hexagonal circle patterns and integrable systems: Patterns with the multi-ratio property and Lax equations on the regular triangular lattice. Int. Math. Res. Notices 2002:3 111-164.

[23] N.P. Dolbilin, A.G. Sedrakyan, M.A. Shtan'ko, M.I. Shtogrin. Topology of a family of parametrizations of two-dimensional cycles arising in the three-dimensional Ising model. Dokl. Akad. Nauk SSSR 295:1 (1987) 19-23 [English translation: Soviet Math. Dokl. 36:1 (1988) 11-15. 
[24] M. Senechal. Quasicrystals and geometry. Cambridge University Press, Cambridge, 1995.

[25] B.G. Konopelchenko, W.K. Schief. Three-dimensional integrable lattices in Euclidean spaces: conjugacy and orthogonality. Proc. R. Soc. A 454 (1998) 3075-3104.

[26] B.G. Konopelchenko, W.K. Schief. Menelaus' theorem, Clifford configurations and inversive geometry of the Schwarzian KP hierarchy. J. Phys. A: Math. Gen. 35 (2002) 6125-6144.

[27] A. King, W. Schief. Tetrahedra, octahedra and cubo-octahedra: integrable geometry of multiratios. J. Phys. A: Math. Gen. 36 (2003) 785802. 